\title{
P- 0615 Frequency, precipitating factors and outcome of Diabetic Ketoacidosis in Type 1 Diabetes Mellitus at Tertiary Care Hospital
}

\author{
Khan, M.I.H.(1)*;Cheema, J.M.(2);
}

Department of Medicine, Division of Diabetes, Endocrine \& Metabolism

Postgraduate Medical Institute/ Ameer Ud Din Medical College,

Lahore General Hospital, Lahore

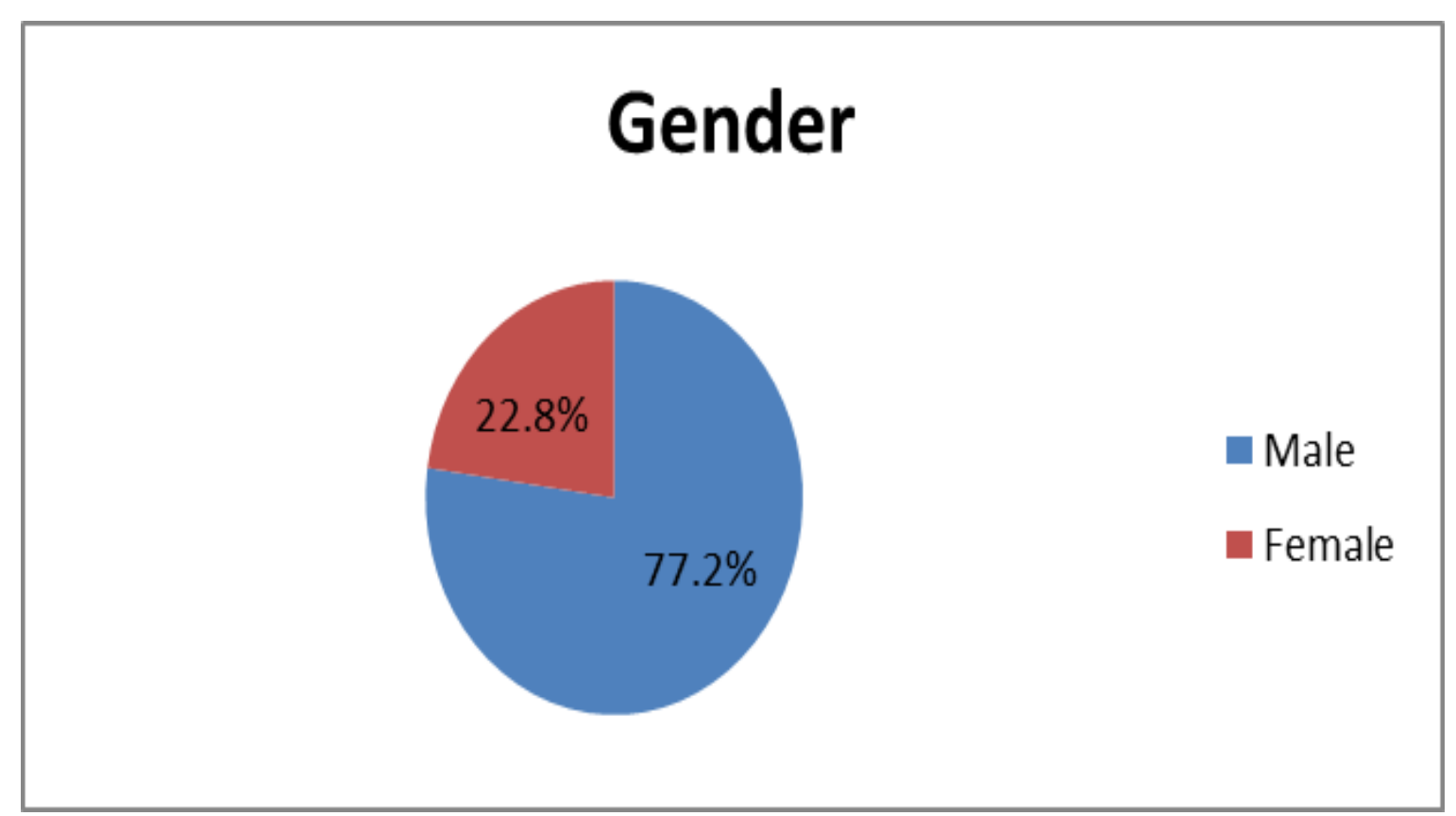

\section{ABSTRACT}

OBJECTIVE:

To observe the frequency, precipitating factors and outcome of diabetic ketoacidosis (DKA) in adults with established Type 1 Diabetes and newly diagnosed Diabetes at a tertiary care hospital.

\section{METHODS:}

Retrospective data of adults admitted with a diagnosis of DKA at LAHORE GENERAL HOSPITAL, Lahore from 1st Jan 2013 till 31st Dec 2015 was collected. The clinical presentations, laboratory investigations, duration of stay in hospital, the treatment required, time of recovery and outcome were compared.

\section{RESULTS:}

A total of 202 patients were included who fulfilled the criteria of DKA. A large number, 160 were $<26$ years of age with a male predominance of 156 . Out of 202 patients, 72 had established Type 1 Diabetes and 130 were newly diagnosed. The commonest presenting complaints in both groups were sepsis (105), missed dose (49) and first presentation (48).

\section{Precipitating Factors For Established and New Cases}
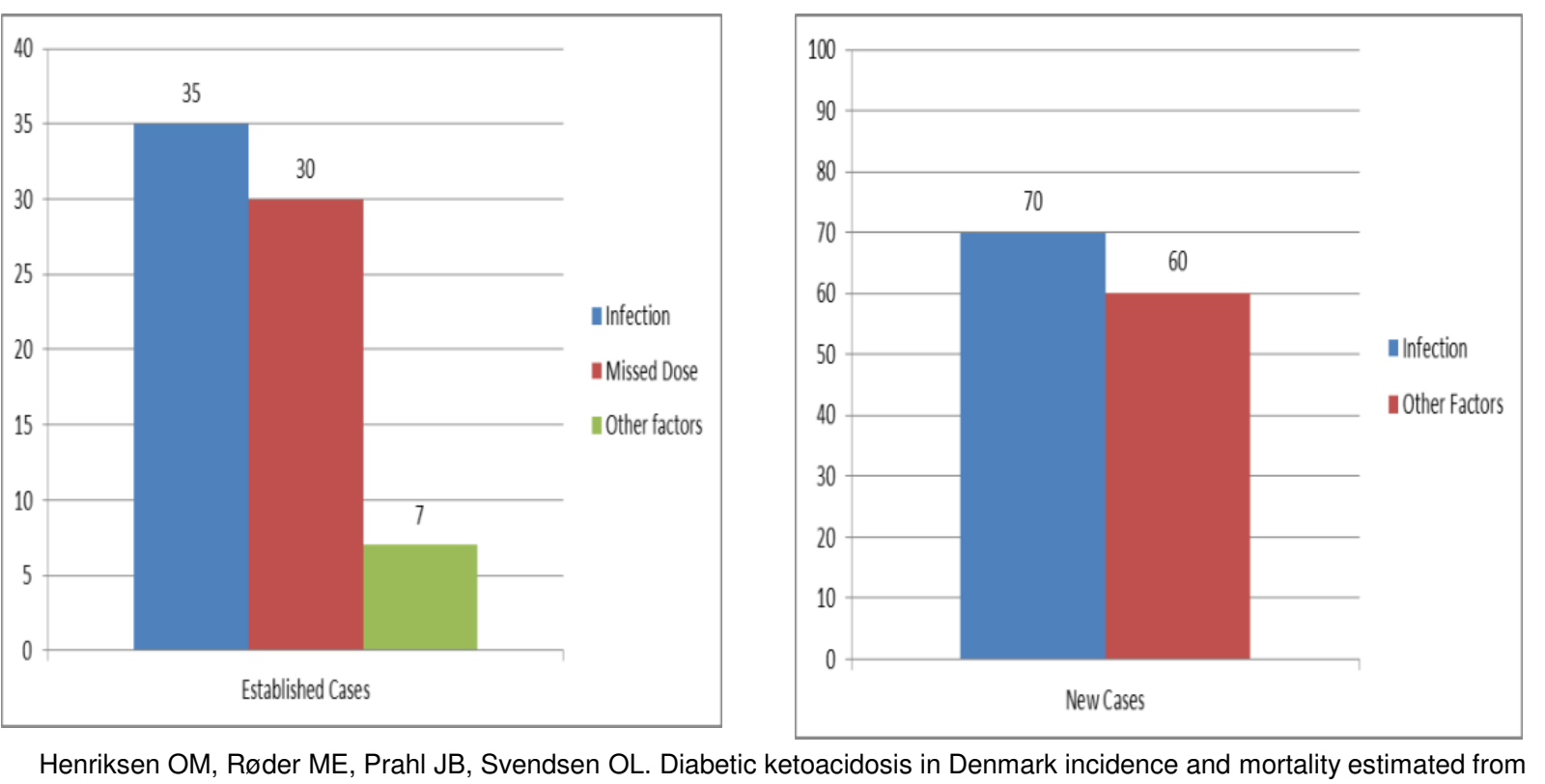

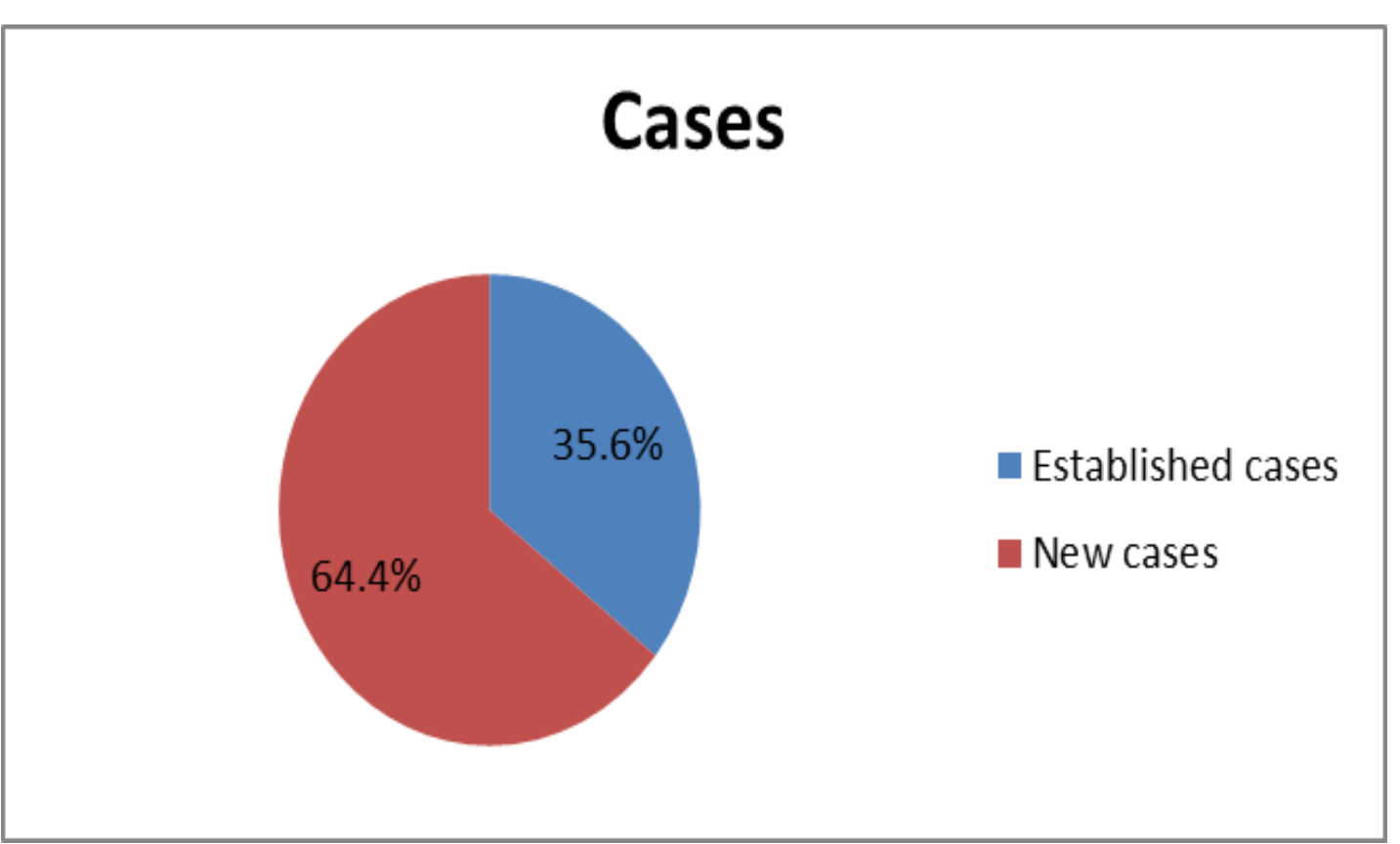

The comparison of clinical improvement and laboratory investigations between the two groups showed that newly diagnosed DM patients had lower $\mathrm{pH}$, bicarbonate and high BSR at presentation as compared to those with established Type $1 \mathrm{DM}$ and patients with established diabetes improved earlier, required lesser duration of intravenous fluids and their insulin was changed to subcutaneous in less time compared with newly diagnosed. Longer stay of more than 7 days was observed in patients with new diagnosis. Mortality was in 15 patients with higher ratio seen in newly diagnosed Type 1.

\section{Outcome of Patients}

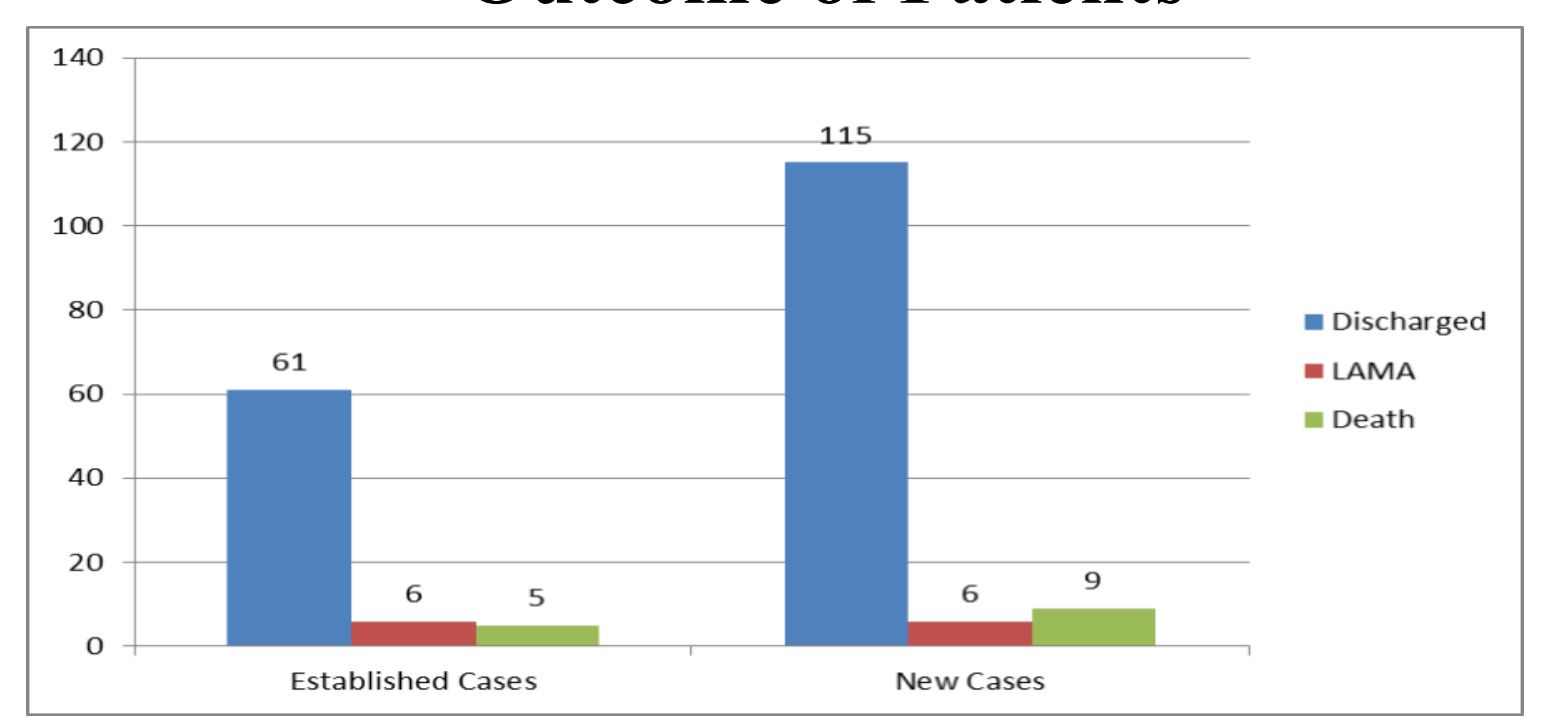

CONCLUSION:

It can be concluded from the above data that earlier diagnosis of Type 1 Diabetes Mellitus, appropriate treatment, regular screening for complications and infections will result in less hospital admissions and better outcome.
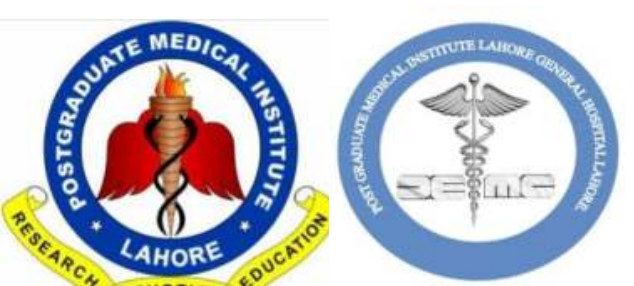